\title{
Dynamics and rigidity of a manipulator with three DOFs
}

\author{
Serikbay Kosbolov*, Yerlan Yeleukulov, Alfiya Atalykova, Algazy Zhauyt, \\ Gulsara Yestemessova, and Saltanat Yussupova \\ Almaty University of Power Engineering and Telecommunication, Almaty 050013, Kazakhstan
}

\begin{abstract}
The problem of dynamic elastic four-link initial kinematic chain (IKC) of the load-bearing manipulator, which is the basis for various modifications are considered. Using the Lagrange operator for this system, equations of motion in matrix form are obtained. To determine the potential energy of an elastic four-link IKC manipulator, we use the formula for the elastic potential energy of a rectilinear homogeneous rod of length $l$. The cross-section of the rod is considered annular or circular. Solving the system of linear equations of motion on a computer using the ADAMS program, the results of the movement of links and cargo were obtained. Kinematics and dynamics are presented for a generic 3 DOFs Initial Kinematic Chain; with anthropometric data and the dynamics equations, simulations were performed to understand its behavior.
\end{abstract}

\section{Introduction}

In the last few years, the number of projects studying the human hand from the robotic point of view has increased rapidly, due to the growing interest in academic and industrial applications. Nevertheless, the complexity of the human hand, given its large number of degrees of freedom (DoF) within a significantly reduced space requires an exhaustive analysis, before proposing any applications [1]. The aim of this paper is to provide a complete summary of the kinematic and dynamic characteristics of the human finger (manipulator) as a preliminary step towards the development of hand devices such as prosthetic/robotic hands and exoskeletons imitating the human hand shape and functionality. Kinematics and dynamics are presented for a generic finger; with anthropometric data and the dynamics equations, simulations were performed to understand its behavior. In the past decade, the applications of intelligent control techniques (fuzzy control or neural- network control) to the motion control of robotic manipulators have received considerable attention [2]. In general, robotic manipulators have to face various uncertainties in their dynamics, such as payload parameter, friction, and disturbance. It is difficult to establish an appropriate mathematical model for the design of a model based control system. Thus, the general claim of these intelligent control approaches is that they can attenuate the effects of structured parametric uncertainty and unstructured disturbance by using their powerful learning ability without a detailed knowledge of the controlled plant in

\footnotetext{
*Corresponding author: kosbolov@mail.ru
} 
the design processes. Feed forward neural networks have been shown to obtain successful results in system identification and control [3]. Such neural networks are static input/output mapping schemes that can approximate a continuous function to an arbitrary degree of accuracy. Results have also been extended to recurrent neural networks [4]. For example, Hamed Khakpour and Lionel Birglen [5] studied the approximation of continuous-time dynamic systems using the dynamic recurrent (DRNN) and a Hopfield-type DRNN was presented by Zhou Ma and Pinhas Ben-Tzvi [6]. As is widely known, both fuzzy logic systems and neural network systems are aimed at exploiting human-like knowledge processing capability. Moreover, combinations of the two have found extensive applications. This approach involves merging or fusing fuzzy systems and neural networks into an integrated system to reap the benefits of both. For instance, H. A. Al-Dois, et al. [7] proposed a general neural network model for a fuzzy logic control and decision system, which is trained to control an unmanned vehicle. In this paper FNN is used to identify and control a three links robot arm. We present the forward and inverse identification as offline learning to use the parameters of this stage in control stage. For control problem, we present the indirect (forward) and direct (inverse) control. Computer simulation implements to view the results of robot arm application.

\section{Materials and methods}

Considering the dynamics of the original kinematic chains (IKC) of a manipulator with three degrees of freedom. Specifically, we will analyze the dynamics of the plane motions of the elastic four-link IKC of the load bearing manipulator, which is the basis for various modifications. [1]. Let the flat IKC consist of three elastic links $A B, B C, C D$, the same length and the same section, and three rotational hinges $A, B, C$ (see Figure 1).

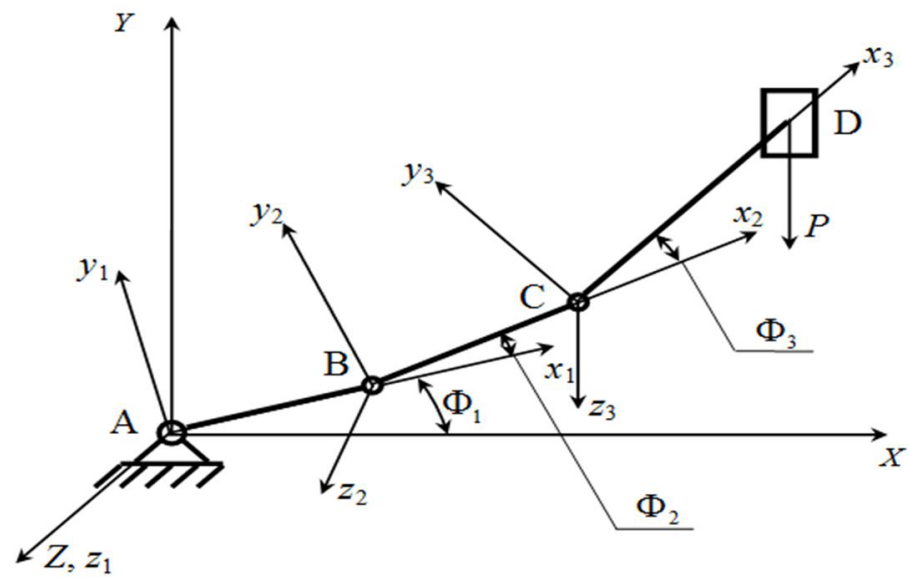

Fig. 1. Initial kinematic chains with the elasticity of links.

Thin elastic rods can perform transverse and torsional oscillations of small amplitude. The change in their length, as well as the elastic longitudinal displacements, is neglected in comparison with the amplitude of the transverse displacements [2]. Cargo $P$ we consider it to be an absolutely rigid body with specified inertial characteristics. Weight $m$, cargo $P$ consider a lot more mass of the IKC. The last assumption allows one to neglect the kinetic energy of the IKC in comparison with the kinetic energy of the cargo, and also to assume that the frequencies of the intrinsic elastic vibrations of the rods are much greater than the vibration frequencies of the load, due to the elasticity of the IKC. 
Table 1. Model parameters.

\begin{tabular}{|c|c|c|c|c|c|c|c|}
\hline № & $\mathbf{A B}, \mathbf{m m}$ & $\mathbf{B C}, \mathbf{m m}$ & $\mathbf{C D}, \mathbf{m m}$ & Rot. Speed & $\mathbf{P}, \mathbf{N}$ & Mass, $\mathbf{k g}$ & Link mass, $\mathbf{k g}$ \\
\hline 1 & 150 & 200 & 100 & 15 & 41.33 & 1 & $\mathrm{~m}_{1}=9$ \\
\hline 2 & 170 & 220 & 110 & 12 & 65.42 & 2 & $\mathrm{~m}_{2}=12$ \\
\hline 3 & 200 & 250 & 125 & 7.5 & 72.37 & 3 & $\mathrm{~m}_{3}=9$ \\
\hline
\end{tabular}

Together with the elastic model, we will also consider an auxiliary absolutely rigid IKC model, in which the angles in the hinges and the lengths of the links are the same as in the real elastic model. Let us find the Lagrange functions for a given system. To determine the potential energy of an elastic three-link IKC, we use the formula for the elastic potential energy of a rectilinear homogeneous rod of length $l$. The cross-section of the rod is considered circular or annular [3]. Let one end, for example, the left one, of the rod be rigidly embedded, and on the other, an elastic displacement vector $\vec{R}$ and the rotation vector $\vec{\Phi}$. The rod is in equilibrium [4]. We denote by $\vec{u}(s)$ vector of the elastic displacement of the neutral axis of the rod, and through $\bar{\varphi}(s)$ angle of rotation of the rod about the axis (here the argument $S$ the length of the rod measured from its left end $0 \prec S \prec l$ ). Potential elastic energy of the deformed rod:

$$
\Pi=\frac{E J}{2} \int_{0}^{l}\left[u^{\prime \prime}(s)\right]^{2} d s+\frac{c}{2} \int_{0}^{l}\left[\varphi^{\prime}(s)\right]^{2} d s
$$

Here the dashes denote the derivatives of $S, E$ modulus of elasticity of rod material, $J$ moment of inertia of the rod section, $c$ - torsional rigidity of the rod [5].

Functions $u(s)$ and $\vec{\varphi}(s)$ in the quasistatic approximation satisfy the boundary conditions:

$$
\begin{gathered}
u^{I V}(s)=0, \quad \varphi^{I I}(s)=0, \quad u(0)=u^{I}(0)=\varphi(0)=0 \\
u(l)=R, \quad u^{I}(l)=\vec{\Phi} \cdot \vec{k}, \quad \varphi(l)=\vec{\Phi} \cdot \vec{k}
\end{gathered}
$$

where $k$ unit vector directed along the axis of the rod in the unreformed state.

Substituting in (1) the solution of the boundary value problem (2) equal to:

$$
\begin{gathered}
\vec{u}(s)=(3 \vec{R}-l \vec{\Phi} \times \vec{k}) \cdot\left(\frac{s}{l}\right)^{2}+(l \vec{\Phi} \times \vec{k}-2 \vec{R}) \cdot\left(\frac{s}{l}\right)^{3} \\
\vec{\varphi}(s)=\Phi \cdot \vec{k}\left(\frac{s}{l}\right)
\end{gathered}
$$

we obtain the potential energy of the elastic rod in the form

$$
\Pi=\frac{2 E J}{l}\left[3 \frac{\vec{R}^{2}}{l^{2}}-3 \frac{\vec{R}}{l}(\vec{\Phi} \times \vec{k})+(\vec{\Phi} \times \vec{k})^{2}\right]+\frac{c}{2 l}(\vec{\Phi} \times \vec{k})^{2}
$$

We will compose the equation of the potential energy of the three-link system using formula (4) and substituting it into place $\vec{R}, \vec{\Phi}$ vectors $\vec{R}_{1}, \vec{\Phi}_{1}, \vec{R}_{2}, \vec{\Phi}_{2}$ accordingly for the first and second rod. We get: 


$$
\begin{gathered}
\Pi=\Pi_{1}+\Pi_{2}=\frac{2 E_{1} J_{1}}{l_{1}}\left[3 \frac{\vec{R}_{1}^{2}}{l_{1}^{2}}-3 \frac{\vec{R}_{1}}{l_{1}}\left(\vec{\Phi}_{1} \times \vec{k}_{1}\right)+\left(\vec{\Phi}_{1} \times \vec{k}_{1}\right)^{2}\right]+\frac{c_{1}}{2 l_{1}}\left(\vec{\Phi}_{1} \times \vec{k}_{1}\right)^{2}+ \\
+\frac{2 E_{2} J_{2}}{l_{2}}\left\{\begin{array}{l}
\frac{3}{l_{2}^{2}}\left(\vec{R}_{2}-\vec{R}_{1}-\vec{\Phi}_{1} \times \vec{k}_{2} l_{2}\right)^{2}-\frac{3}{l_{2}}\left(\vec{R}_{2}-\vec{R}_{1}-\vec{\Phi}_{1} \times \vec{k}_{2} l_{2}\right) \cdot\left[\left(\vec{\Phi}_{2}-\vec{\Phi}_{1}\right) \times \vec{k}_{2}\right]+ \\
+\left[\left(\vec{\Phi}_{2}-\vec{\Phi}_{1}\right) \times \vec{k}_{2}\right]^{2}
\end{array}\right\}+\frac{c_{2}}{2 l_{2}}\left[\left(\vec{\Phi}_{2}-\vec{\Phi}_{1}\right) \times \vec{k}_{2}\right]^{2}(5)
\end{gathered}
$$

where $l_{j}(j=1,2)$ length of the IKC links, $c_{j}(j=1,2)$ bending stiffness, $k_{j}(j=1,2)$ torsional stiffness of links [6].

In view of the assumptions made, the kinetic energy of the system is the kinetic energy of the plane motion of the cargo $P$ is equal to:

$$
T=0,5 m \dot{r}_{Q}^{2}+0,5 J\left(\dot{\varphi}_{1}+\dot{\varphi}_{2}+\dot{\varphi}_{3}+\dot{\psi}\right)^{2}
$$

where

$$
\begin{gathered}
\dot{\vec{r}}_{Q}^{2}=\dot{\vec{r}}_{x}^{2}+\dot{\vec{r}}_{y}^{2} \\
r_{x}=l_{1} \cos \varphi_{1}+\left(l_{2}+x\right) \cos \left(\varphi_{1}+\varphi_{2}\right)-y \sin \left(\varphi_{1}+\varphi_{2}\right)+r\left[\cos \left(\varphi_{1}+\varphi_{2}+\varphi_{3}\right)-\psi \sin \left(\varphi_{1}+\varphi_{2}+\varphi_{3}\right)\right] \\
r_{y}=l_{1} \sin \varphi_{1}+\left(l_{2}+x\right) \sin \left(\varphi_{1}+\varphi_{2}\right)+y \cos \left(\varphi_{1}+\varphi_{2}\right)+r\left[\sin \left(\varphi_{1}+\varphi_{2}+\varphi_{3}\right)+\psi \cos \left(\varphi_{1}+\varphi_{2}+\varphi_{3}\right)\right]
\end{gathered}
$$

Let us compose the equations of motion of the IKC. We assume that only the control actions in the hinges act on the IKC with the load, which ensure the variation of the angles according to given laws: $\varphi_{j}=\varphi_{j}(t)(j=1,2,3)$.

We use the Lagrange operator:

$$
\frac{d}{d t} \frac{\partial L}{\partial q_{j}}-\frac{\partial L}{\partial q_{j}}=0(j=1,2,3)
$$

in which we substitute $L=T-\Pi^{\prime}$ according to (6.6) - (6.7).

$$
\begin{gathered}
\Pi^{\prime}=\frac{2 E_{1} J_{1}}{l_{1}}\left[3 \frac{\left(\vec{R}_{1}^{\prime}\right)^{2}}{l_{1}^{2}}-3 \frac{R_{1}^{\prime}}{l_{1}}\left(\vec{\Phi}_{1}^{\prime \prime} \times \vec{k}_{1}\right)+\left(\vec{\Phi}_{1}^{\prime \prime} \times \vec{k}_{1}\right)^{2}\right]+ \\
+\frac{2 E_{2} J_{2}}{l_{2}}\left\{\begin{array}{l}
\frac{3}{l_{2}^{2}}\left(\vec{R}_{2}^{\prime}-\vec{R}_{1}^{\prime}-\vec{\Phi}_{1}^{\prime \prime} \times \vec{k}_{2} l_{2}\right)^{2}-\frac{3}{l_{2}}\left(\vec{R}_{2}^{\prime}-\vec{R}_{1}^{\prime}-\vec{\Phi}_{1}^{\prime \prime} \times \vec{k}_{2} l_{2}\right)\left[\left(\vec{\Phi}_{2}^{\prime \prime}-\vec{\Phi}_{1}^{\prime \prime}\right) \times \vec{k}_{2}\right]+ \\
+\left[\left(\vec{\Phi}_{2}^{\prime \prime}-\vec{\Phi}_{1}^{\prime \prime}\right) \times \vec{k}_{2}\right]^{2}
\end{array}\right\}
\end{gathered}
$$

where $\Pi^{\prime}$ potential energy corresponding to motions in the plane Oxy.

After this, the equations of motion in the matrix form take the form:

$$
A \ddot{q}+G \dot{q}+C q=B \ddot{\varphi}+f
$$

where $A, G, C$, In the matrix size $3 \times 3, q, \varphi, f$ vector columns, matrices $A, C, B$ depend only on the configuration of the IKC. 
After the transformations, we obtain a linear system equation of motion of the form:

$$
\ddot{q}=G_{1} q+C_{1} q=B_{1} \ddot{\varphi}+f_{1}
$$

where $q$ vector with components $x^{\prime}, y^{\prime}, \psi$.

We omit the strokes of the dimensionless variables $y$, and the matrices $G_{1}, C_{1}, B_{1}$ and vector $f_{1}$ are obtained from matrices $A, G, C, B$ conversion result [7]. As a result, we obtain a system (10) containing dimensionless parameters:

$$
\xi=\left[\frac{E_{2} I_{2}}{E_{1} I_{1}}\right] \eta, \quad \eta=\frac{l_{2}}{l_{1}}, \quad \chi=\frac{r}{l_{2}}, \quad \beta=\frac{m l_{2}^{2}}{I}
$$

Under zero initial conditions $x(0)=y(0)=\dot{x}(0)=\dot{y}(0)=\psi(0)=\dot{\psi}(0)=0 \quad$ it is possible to integrate a linear system of equations of motion on a computer on the interval $[0$, $\tau]$, which will show that the movement of the load is of an oscillating nature, and the frequency of the oscillations of the cargo varies markedly with the angle $\varphi_{2}$.

\section{Results and discussion}

The two-dimensional static stiffness of the index finger was measured with the interphalangeal joints in flexed and extended postures. The stiffness of the relaxed finger was compared with the stiffness when voluntary force was exerted in different directions. The finger stiffness was found to be anisotropic, with the direction of greatest stiffness being approximately parallel to the proximal phalange of the finger. This direction was relatively unaffected by finger posture or direction of finger force. Finger stiffness was more anisotropic when the interphalangeal joints were extended than flexed. The stiffness was most anisotropic when the interphalangeal joints were extended and force was being exerted in the direction of pointing, while it was least anisotropic when the interphalangeal joints were flexed and force was being exerted in directions normally associated with pinching and tapping actions. The stiffness of the individual finger joints was computed and the relation between stiffness and joint torque was examined. Previous studies, which examined single finger joints in isolation, had found that joint stiffness varied in a linear fashion with net joint torque. In contrast, we did not find a monotonic relation between joint stiffness and net joint torque, which we attributed to the need to vary the amount of cocontraction of antagonistic muscles when controlling the direction of finger force. The performed calculations showed that:

The results of the study are shown accordingly $\Phi_{1}=15^{\circ}$ at $t_{1}=0.015 \mathrm{~s}$ then, the value maximum Strain-Energy $[118.3769 ; 118.4457 ; 118.6521] \mathrm{N} * \mathrm{~mm}$, if $\Phi_{2}\left[10^{\circ} ; 15^{\circ} ; 30^{\circ}\right]$ and $\left[t_{1}=0.015 ; t_{2}=0.78 ; t_{3}=1.335\right] \mathrm{s}$ is equal, maximum Strain-Energy [61.3341; $120.9643 ; 86.4937] \mathrm{N} * \mathrm{~mm}$ amount, $\Phi_{3}\left[5^{\circ} ; 15^{\circ} ; 45^{\circ}\right]$ and $\left[t_{1}=0.75 ; t_{2}=2.745 ; t_{3}=1.38\right.$ ] s are equal, the results obtained showed maximum Strain-Energy [8.121;40.1519;28.5888] $\mathrm{N} *$ mm (see Fig. 3-5). 


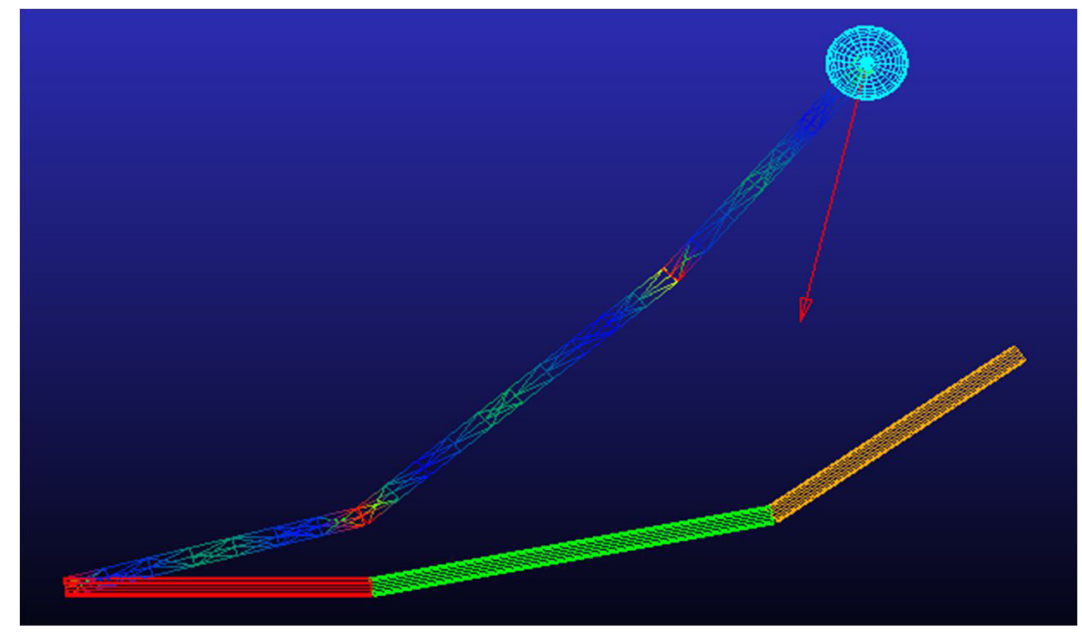

Fig. 2. Model of MSC ADAMS 3 DoFs mechanism.
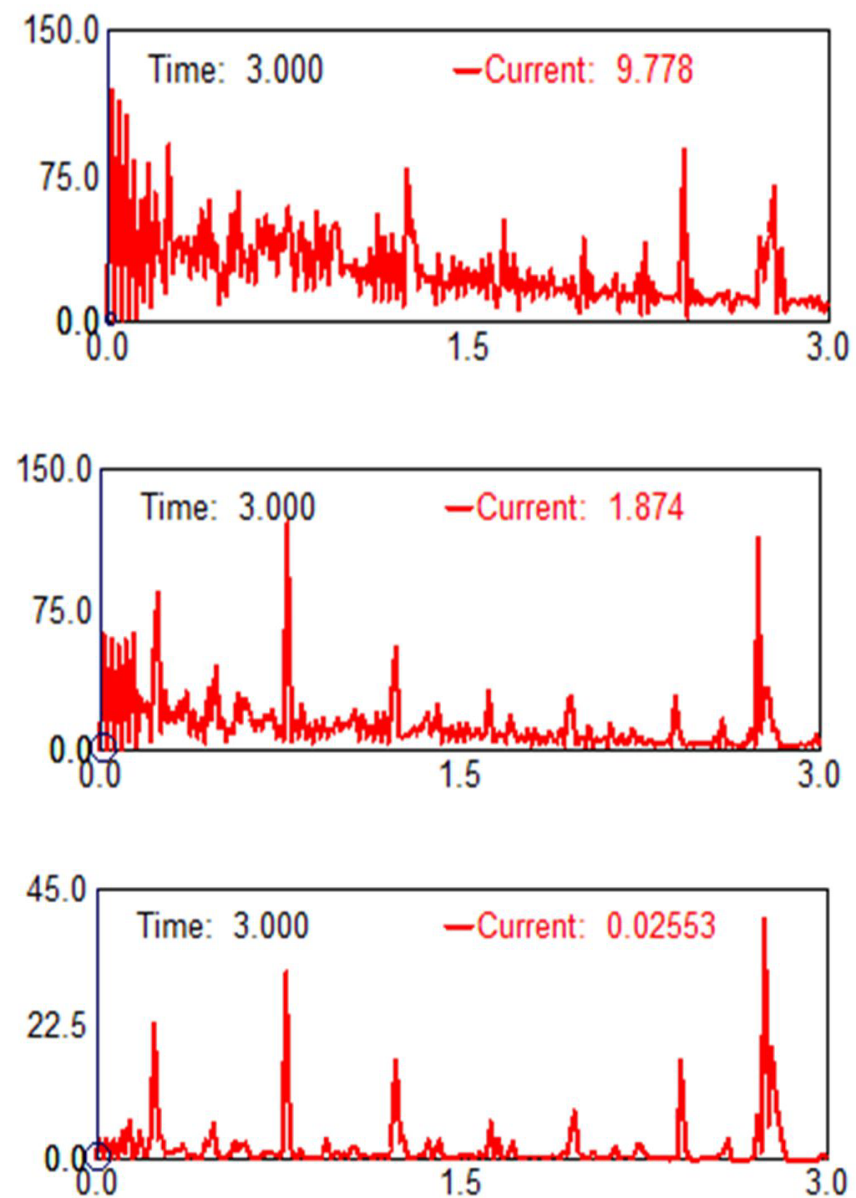

Fig. 3. Computed plot of Strain-Energy AB, BC, CD $\left(\phi_{1}=\phi_{2}=\phi_{3}=15^{\circ}\right)$. 

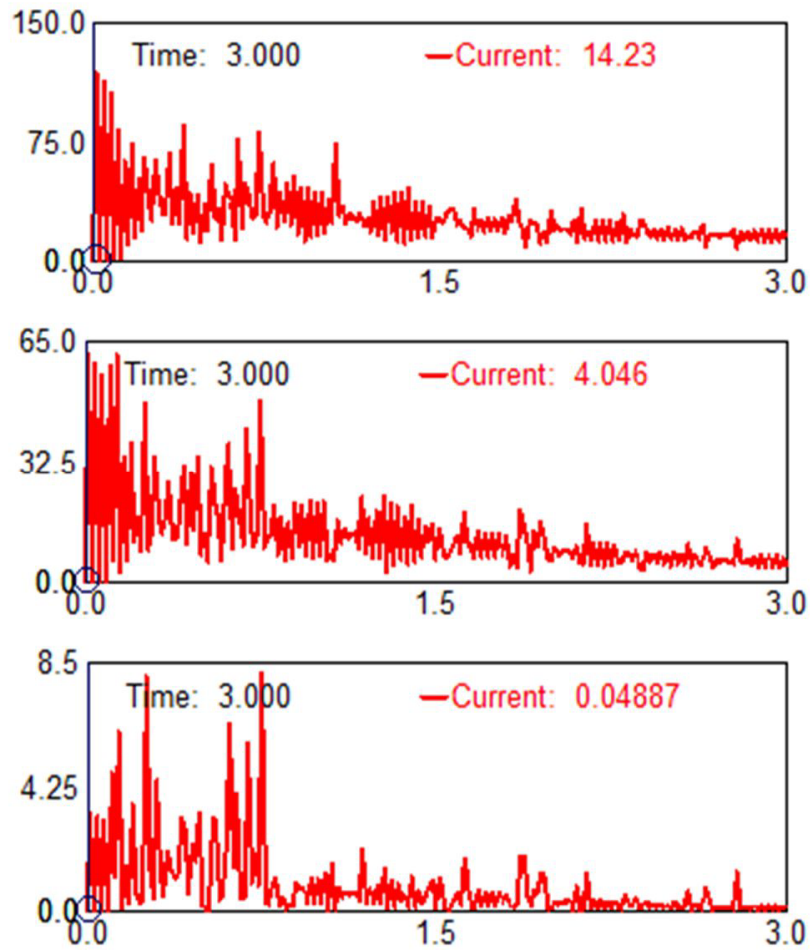

Fig. 4. Computed plot of Strain-Energy AB, BC, CD $\left(\phi_{1}=15^{\circ}, \phi_{2}=10^{\circ}, \phi_{3}=5^{\circ}\right)$.
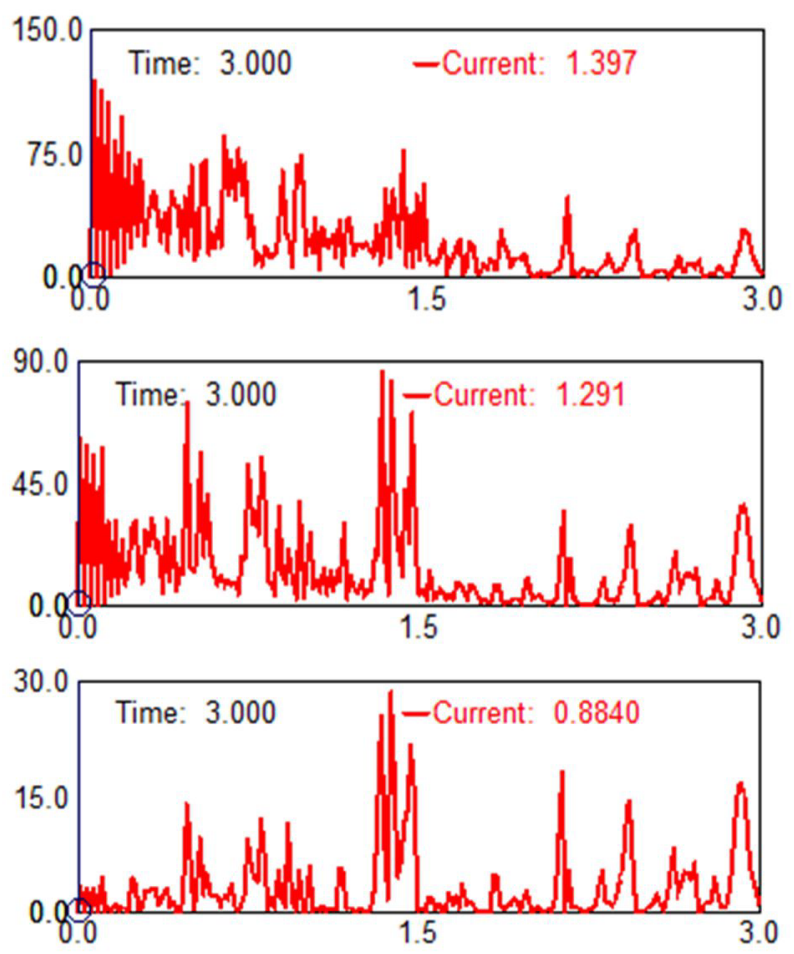

Fig. 5. Computed plot of Strain-Energy AB, BC, CD $\left(\phi_{1}=15^{\circ}, \phi_{2}=30^{\circ}, \phi_{3}=45^{\circ}\right)$. 


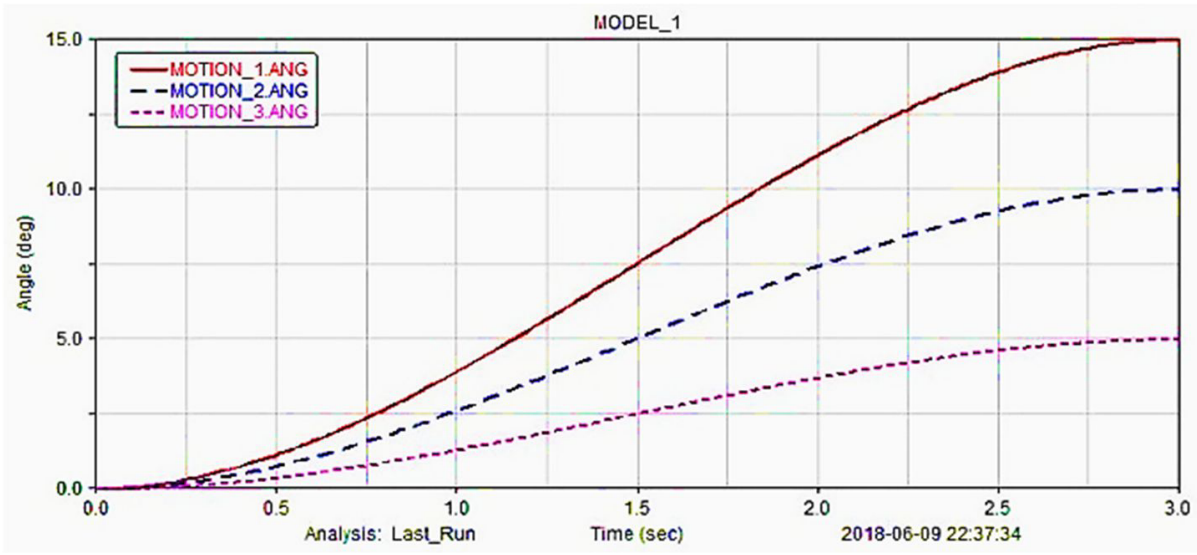

a)

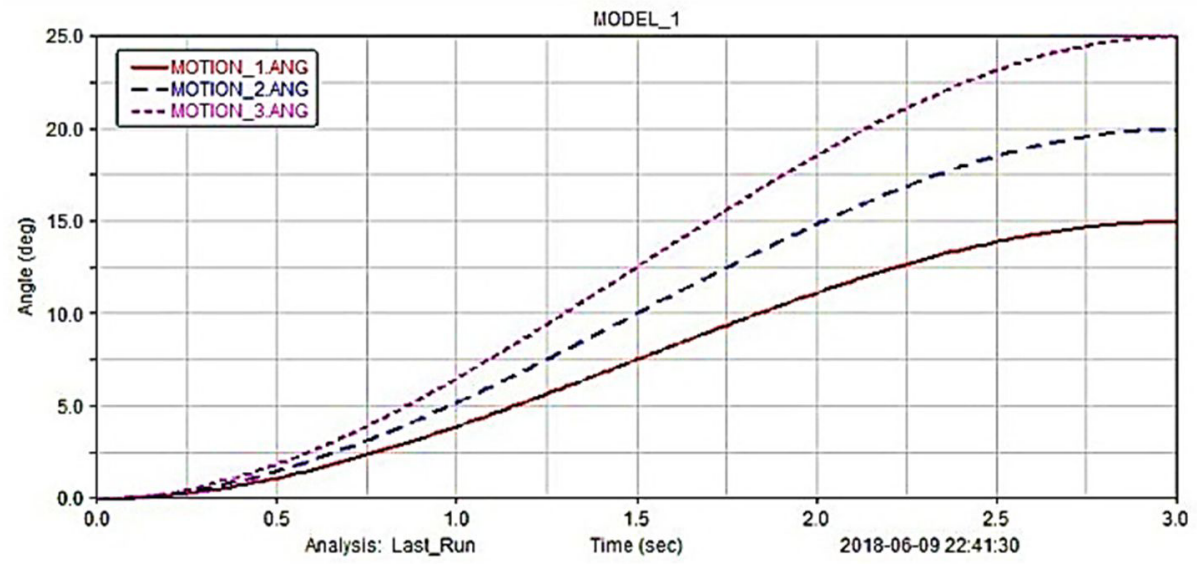

b)

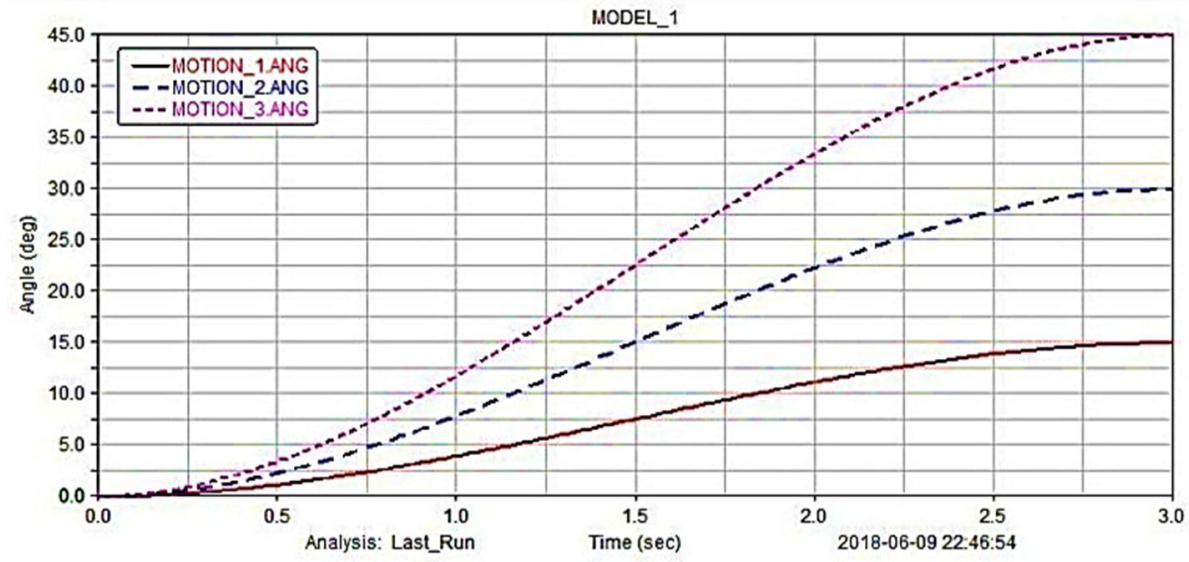

c)

Fig. 6. Behavior of the joint angles in time:

a) step $\phi_{1}=15^{\circ}, \phi_{2}=10^{\circ}, \phi_{3}=5^{\circ}$; b) step $\phi_{1}=15^{\circ}, \phi_{2}=20^{\circ}, \phi_{3}=25^{\circ}$; c) step $\phi_{1}=15^{\circ}, \phi_{2}=30^{\circ}, \phi_{3}=45^{\circ}$. 


\section{Conclusions}

The peculiarities of compiling dynamic models of non-closed four-link initial kinematic chains of the manipulator are considered taking into account the elasticity of the links. A system linear equations of motion in matrix form is obtained, the solution of which showed that the movement of the load is of an oscillating nature, and the frequency oscillations of the load changes markedly with the angle $\varphi_{2}$.

\section{References}

1. F. C. Chen, S. Appendino, A. Battezzato, A. Favetto, M. Mousavi, F. Pescarmona Mechanisms and Machine Science, 17, 115 (2014)

2. J. A. Snyman, L. J. du Plessis, J. Duffy, J. Mech. Des, 122 (4), 447 (2000)

3. B. Belzile, L. Birglen, J. Mechanisms Robotics, 9 (5), 051004 (2017)

4. Y. J. Shin, H. J. Lee, K. S. Kim, S. Kim, Journal of mechanical engineering and automation, 2 (5), 85 (2012)

5. H. Khakpour, L. Birglen, J. Comput. Nonlinear Dynam, 8 (2), 021010 (2012)

6. Z. Ma, P. Ben-Tzvi, J. Mechanisms Robotics, 7 (4), 041008 (2015)

7. H. A. Al-Dois, A. K. Jha, R. B. Mishra, Journal of The Institution of Engineers, 93, 3, 257 (2012) 